\title{
ANÁLISE DE REGRESSÃO E PREVISÃO DOS DISPÊNDIOS DO GOVERNO FEDERAL EM CIÊNCIA, TECNOLOGIA E INOVAÇÃO
}

\author{
R.G. Cordeiro ${ }^{1}$; M.S. Mussa ${ }^{\text {* }}$; H.R.M. Hora ${ }^{1}$, A.C. Morais ${ }^{1}$ \\ 1 Instituto Federal Fluminense, 28080-565, Campos dos Goytacazes-RJ, Brasil \\ *munirmussa@gmail.com
}

\section{RESUMO}

A inovação tem sido reconhecida, cada vez mais, como um dos fatores que possuem forte impacto positivo para o desenvolvimento econômico e fortalecimento da competitividade. Dar conhecimento e divulgar informações que venham a contribuir para um melhor entendimento sobre as diversas formas de incentivo, sobretudo governamental, são de vital importância para a geração de novos conhecimentos e de inovação tecnológica. O presente artigo tem o objetivo de realizar a previsão dos investimentos em ciência e tecnologia (C\&T) despendidos pelo governo federal para o período de 2014 a 2018. Para isso, utilizando-se do software CurveExpert e da técnica de mineração de dados conhecida como regressão, que visa prever valores numéricos, são analisados dados dos investimentos federais disponíveis entre os anos de 2000 a 2013. A partir dos gráficos gerados e da técnica utilizada foi possível obter como resultado uma análise do comportamento dos investimentos do governo federal no período de 2000 a 2018, ficando visíveis os períodos de crescimento, de baixas taxas e estabilidade. Concluindo assim que, o cálculo para o período de 2014 a 2018 é de aumento nos investimentos do governo federal em duas das instituições abordadas no estudo e leve queda para outras duas.

PALAVRAS-CHAVE: Pesquisa e Desenvolvimento; Mineração de dados; Análise de regressão; Inovação.

\section{REGRESSION ANALYSIS AND PREVISION OF PUBLIC SPENDINGS AT FEDERAL GOVERNMENT'S SCIENCE, TECHNOLOGY AND INNOVATION}

\begin{abstract}
Innovation has been increasingly recognized as one of the factors that have a strong positive impact on economic development and competitiveness. Giving knowledge and disseminating information that will contribute to a better understanding of the various forms of incentive, especially governmental, are of vital importance for the generation of new knowledge and technological innovation. The objective of this article is to prevision the investments in science and technology expended by the federal government for the period from 2014 to 2018. For this purpose, using CurveExpert software and the data mining technique known as
\end{abstract}

regression, which aims to predict numerical values, data from federal investments available between 2000 and 2013 are analyzed. Based on the graphs generated and the technique used, it was possible to obtain an analysis of the behavior of federal government investments in the period 2000 to 2018, showing periods of growth, low rates and stability. Concluding, therefore, the calculation for the period from 2014 to 2018 is an increase in federal government investments in two of the institutions addressed in the study and slight decrease for two others. 
KEYWORDS: Research and Development; Data mining; Regression analysis; Innovation. 


\section{INTRODUÇÃO}

Ao longo da história, as inovações tecnológicas e as invenções sempre foram vistas com alguma desconfiança pela humanidade, muito em função da falta de percepção de vantagem nelas. Essas resistências, no entanto, tendiam a desaparecer quando as pessoas começavam a identificar tais vantagens materiais, econômicas ou, até mesmo, militares nas novas ideias. Complementarmente, foram gerados mais investimentos por parte dos governos, assim como gastos individuais em pesquisas aplicadas. Sobretudo em países desenvolvidos, foi implementada a aplicação do conhecimento científico como forma de fomentar o desenvolvimento tecnológico e a inovação. As atividades de P\&D seriam, então, o fator iniciador e o indicador fundamental da inovação (MATIAS-PEREIRA, 2011).

Em um ambiente econômico baseado no conhecimento e caracterizado pela existência de mercados dinâmicos e competitivos, surge um conceito para ultrapassar os desafios da busca constante pela inovação. Utilizando-se da teoria da Tríplice hélice, que tem como base a relação entre três esferas institucionais, envolvendo a universidade, a empresa e o governo, verifica-se a necessidade de cada hélice realizar o seu papel de maneira bem definida. Nesse contexto o papel do governo é bem claro: criar mecanismos para promover novas estruturas organizacionais, desenvolvimento econômico, áreas de atuação, bem como conduzir os processos de mudança. Essa relação busca, de uma forma mais eficiente, a produção de novos conhecimentos, a inovação tecnológica e o desenvolvimento econômico (ETZKOWITZ; LEYDESDORFF, 2000; THERGBRAZIL, 2013).

Luna et al. (2008) afirmam que a literatura acadêmica aponta a quantidade de investimento realizado como um fator fundamental para o crescimento econômico sustentável, sobretudo no longo prazo. Portanto, tanto a disponibilidade de recursos quanto a eficiência em sua utilização são fatores preponderantes. De acordo com a Pesquisa Industrial de Inovação Tecnológica (Pintec) comparando o percentual de empresas industriais inovadoras que utilizaram ao menos um instrumento de apoio governamental no período 2006 a 2008 com o resultado observado no período 2009 a 2011, verifica-se que houve aumento nessa participação: de 22,8\% para 34,6\%. Esse representa cerca de 14,3 mil empresas industriais que utilizaram algum incentivo público federal para desenvolver suas inovações de produto e/ou processo nesse último período pesquisado. Para Luna et al. (2008) isso pode ocorrer por falta de conhecimento por parte das empresas sobre os investimentos do governo.

Nesse contexto, com o intuito de dar conhecimento e analisar os investimentos do governo federal nesta área, este trabalho justifica-se pela importância da aplicação de recursos como fonte de promoção da inovação no país. Além disso, a análise dos investimentos do governo federal representa grande importância devido a essa fonte de investimento representar uma parte considerável dos valores despendidos em ciência, tecnologia e inovação no Brasil, conforme dados do Ministério da Ciência, Tecnologia e Inovação (2015).

O presente artigo tem o objetivo de analisar os investimentos em ciência e tecnologia (C\&T) despendidos pelo governo federal entre os anos de 2000 a 2013 e realizar previsão de investimentos para os cinco anos seguintes. Através do estudo realizado é possível obter uma visão do comportamento do investimento do governo federal entre o período dos anos 2000 a 2018 obtendose uma opção visual sobre tais investimentos.

\section{REVISÃO BIBLIOGRÁFICA}

Nesta seção são apresentados os temas que são abordados nesta pesquisa e os trabalhos que colaboraram para a realização desta. 


\subsection{Descoberta de conhecimento em base de dados}

O avanço das novas tecnologias de informação, a utilização e capacidade de armazenamento em bancos de dados estão em constante crescimento. Consequentemente, a atividade de extrair informações relevantes dessas bases é uma tarefa cada vez mais complexa (WITTEN; FRANK, 2005). Nesse sentido, de acordo com Santos, Menezes e Hora (2014), surge o processo de seleção de dados, chamado de Knowledge Discovery in Databases - KDD, que significa Descoberta de conhecimento em base de dados.

Resumidamente Fayyad, Piatetsky-Shapiro e Smyth (1996) definem o KDD como "o processo não trivial de identificação de padrões válidos, novos, potencialmente úteis e, em última instância, compreensíveis em dados". O processo pode ser definido como a descoberta de padrões, relacionamentos e tendências significativas por meio de uma cuidadosa análise dos grandes conjuntos de dados armazenados.

Muitos autores consideravam sinônimos os termos $K D D$ e Mineração de Dados, no entanto, o primeiro termo refere-se ao processo de descoberta de conhecimento útil de um banco de dados como um todo, enquanto mineração de dados está inserida como um dos principais passos necessários para a realização do processo de KDD (CARDOSO; MACHADO, 2008; FAYYAD; PIATETSKY-SHAPIRO; SMYTH, 1996). O processo de descoberta de conhecimento em banco de dados é constituído das seguintes etapas ilustradas na Figura 1.

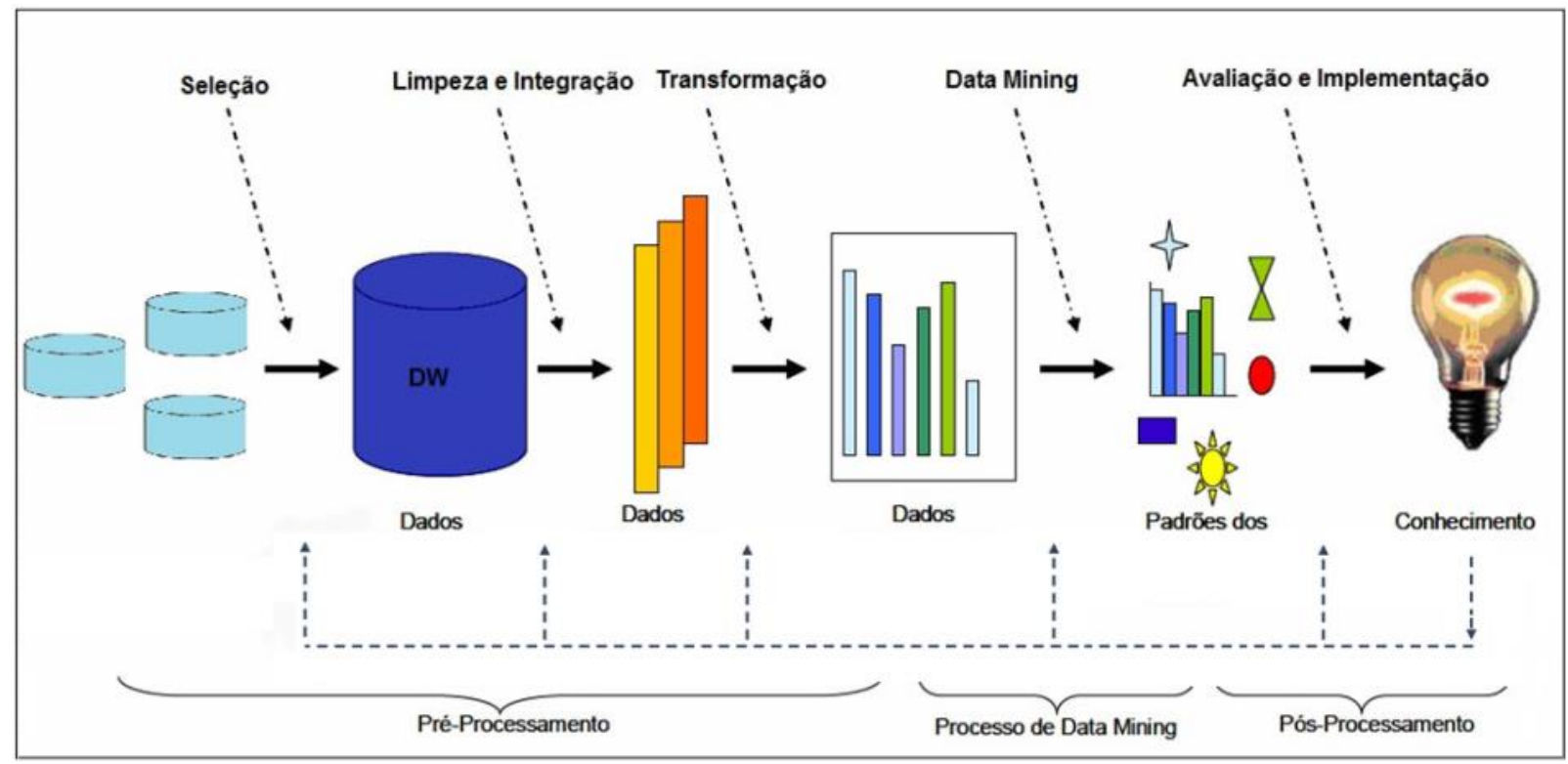

Figura 1 - Etapas do Processo de Descoberta de Conhecimento em Banco de Dados.

Fonte: (SILVA et al., 2010 adaptado de FAYYAD; PIATETSKY-SHAPIRO; SMYTH, 1996).

As etapas do processo de KDD são descritas por Goldschmidt e Passos (2005):

a) Seleção de dados: identificar e selecionar quais informações, dentre as bases de dados existentes, devem ser efetivamente consideradas durante o processo;

b) Limpeza de dados: Realizar tratamento sobre os dados, a fim de assegurar a qualidade relacionada à completude, veracidade e integridade, ou seja, dados inconsistentes ou fora dos padrões são removidos;

c) Integração de dados: Reunir várias fontes de dados, mantendo a consistência e a coerência dos dados integrados; 
d) Transformação de dados: Codificar os dados para o formato apropriado para a próxima etapa. Esta fase é realizada dependendo dos algoritmos que será aplicado na mineração de dados;

e) Data Mining (Mineração de Dados): Aplicar métodos com o propósito de extrair os padrões de interesse;

f) Avaliação de padrões: Identificar os padrões de interesse de acordo com algum critério do usuário;

g) Apresentação de conhecimento: Tornar o conhecimento extraído compreensível ao homem através de gráficos, diagramas ou relatórios demonstrativos.

São chamadas mineração de dados, portanto, todas as técnicas que permitem extrair, analisar e explorar conhecimento de uma massa de dados em busca de padrões, erros e associações que, de outra maneira, permaneceria escondido nas grandes bases (AMARAL, 2016).

Cardoso e Machado (2008) corroboram acrescentando que a mineração dos dados consiste em buscar padrões consistentes para valores pré-determinados através de modelos sofisticados. Ou seja, explorar e analisar por meios automáticos ou semiautomáticos um grande volume de dados e realizar combinações de acordo com valores anteriormente especificados.

A mineração de dados tem sua aplicação cada vez mais difundida em diversas áreas, então são necessárias algumas etapas para sua execução. Após a especificação do que se busca nos dados, que tipo de regularidades ou categorias de padrões interessa, deve ser utilizada uma técnica que garantam como identificar tais padrões da melhor maneira: classificação, associação, agrupamento, regressão e previsão. Definindo-se a tarefa e a técnica, opta-se por uma ferramenta disponível no mercado capaz de facilitar a extração do conhecimento. Alguns dos maiores fornecedores comerciais são: Microsoft, SAS, IBM e Oracle. Dentre ferramentas Open Source mais populares tem-se: Weka, R-Project e Orange (AMARAL, 2016).

\subsection{Pesquisa \& desenvolvimento, Ciência \& tecnologia e inovação}

Calmanovici (2011) afirma que atualmente no Brasil a ciência e tecnologia fazem, cada vez mais, parte do cotidiano das pessoas através da inovação. O autor exemplifica citando o etanol biocombustível e um tratamento de saúde especializado. A proximidade da tecnologia no nosso cotidiano está diretamente relacionada ao esforço em inovação e o estabelecimento de uma cultura inovadora incentivando o esforço em $\mathrm{P} \& \mathrm{D}$ nas empresas. Para que isso seja possível, é necessário contar com a mobilização de diversos setores da sociedade, dentre eles: o investimento público, privado, nacional ou internacional, em empresas que tenham competência em desenvolver e oferecer produtos e serviços inovadores e competitivos, e que também saibam como trabalhar o conhecimento acadêmico e científico transformando-o em tecnologias com retorno para a sociedade. A inter-relação desses fatores é o elemento fundamental desse processo de construção sustentável para a inovação.

Para Tidd e Bessant (2013) "a inovação é movida pela habilidade de estabelecer relações, detectar oportunidades e tirar proveito delas". Para o autor a inovação não tem importância apenas no empreendimento individualizado, mas também como fonte principal do crescimento econômico em nível nacional. E está se transformando no elemento central de políticas econômicas nacionais sendo crescente o conjunto de medidas políticas destinadas a incentivar e promover a inovação. Sendo as pesquisas financiadas em universidades um meio de promover os estudos na área de ciência e tecnologia e uma fonte e informação para tomada de decisões das empresas sobre quando e se utilizar uma nova tecnologia. 
A inovação está diretamente ligada a competitividade, uma vez que, na sociedade brasileira, as limitações em estimular plenamente a inovação, nas empresas particularmente, subtraem competitividade ao país além de representar obstáculo a um maior desenvolvimento econômico. Nesse sentido é fundamental adotar uma política industrial, focada em longo prazo, na qual a inovação tenha destaque para, assim, garantir o desenvolvimento econômico e social. "A busca por novos modelos, instrumentos e arranjos organizacionais é essencial para garantirmos a consolidação do existente e a evolução para uma liderança brasileira em inovação" (CALMANOVICI, 2011). Portanto, acrescenta o autor, a capacidade de inovação é determinante para a competitividade das empresas e das nações como um todo, sobretudo em um mundo cada vez mais globalizado.

No Brasil, a inovação está inserida na política de Ciência e Tecnologia (C\&T), esta é formulada e implementada pelo Conselho Nacional de Ciência e Tecnologia (CCT) responsável por formular as diretrizes da política nacional de desenvolvimento científico e tecnológico.

\subsection{A tríplice hélice}

A abordagem da Hélice Tríplice foi desenvolvida por Henry Etzkowitz e Loet Leydesdorff, e baseia-se na perspectiva da Universidade como indutora das relações com as empresas e o governo. A relação busca a produção de novos conhecimentos, a inovação tecnológica e o desenvolvimento econômico. Nesse contexto, a inovação é compreendida como o resultado de um processo complexo e dinâmico de experiências nas relações entre ciência, tecnologia, pesquisa e desenvolvimento geradas nas universidades, empresas e nos governos (THERG-BRAZIL, 2013).

Nessa abordagem, cada hélice tem o seu papel bem definido, à universidade tem a tarefa de criar fontes de novos conhecimentos e tecnologias, estabelecer relações entre as empresas e os governos, criar novas áreas de atuação, bem como conduzir os processos de mudança. Ao governo cabe promover o desenvolvimento econômico e social através de novas estruturas organizacionais, planos políticos com metas objetivas e voltadas para inovação e conhecimento. Enfim, às empresas cabe desenvolver produtos e serviços inovadores, promover a interação com os centros de transferência de tecnologia da comunidade científica e guiar os processos de mudança (PEREIRA NETO; GALLINDO; CRUZ, 2004).

$\mathrm{O}$ argumento tem base na relação entre a Universidade e a Sociedade na qual Etzkowitz e Leydesdorff (2000) afirmam que a universidade estaria vivendo uma "Segunda Revolução Acadêmica", marcada pela forte sinergia entre instituições acadêmicas e empresas. A Universidade incorpora, além do ensino e da pesquisa, uma terceira missão que é ser um ator ativo do desenvolvimento econômico para a geração de conhecimento científico e tecnológico e, consequentemente, inovação. Nessa abordagem, os grupos de pesquisa das universidades atuam como quase-firmas e interagem com os atores das demais hélices. O resultado é uma Universidade Empreendedora onde o relacionamento entre as hélices assume diferentes configurações: organizações de intermediação são estruturadas como incubadoras e parques tecnológicos; escritórios de propriedade intelectual e comercialização de tecnologia; redes de conhecimento; arranjos produtivos locais (APLs); e universidades corporativas entre outros (THERG-BRAZIL, 2013).

Segundo Webster e Etzkowitz (1991 apud DAGNINO, 2009), entre as razões que explicam a ampliação das relações Universidade-Empresa (U-E) estariam, do lado das empresas:

- custo crescente da pesquisa e desenvolvimento de produtos e serviços necessários para assegurar posições vantajosas no mercado competitivo;

- a necessidade de compartilhar o custo e o risco das pesquisas pré-competitivas com outras instituições que dispõem de suporte financeiro governamental; 
- elevado ritmo no surgimento de inovações no setor produtivo e a redução do intervalo de tempo entre a obtenção dos primeiros resultados de pesquisa e sua aplicação;

Sob a ótica da universidade, as motivações principais seriam:

- a dificuldade crescente para obtenção de recursos públicos para a pesquisa universitária e a expectativa de que estes possam ser proporcionados pelo setor privado em função do maior potencial de aplicação de seus resultados na produção;

- interesse da comunidade acadêmica em legitimar seu trabalho junto à sociedade que é, em grande medida, a responsável pela manutenção das instituições universitárias.

A ampliação dessas relações estaria apontando para uma maior eficiência da relação Universidade-Empresa-Governo, fortemente ligada as relações entre a universidade e seu entorno que estariam levando a universidade a incorporar funções de desenvolvimento econômico às suas tradicionais atividades de ensino. A pesquisa universitária pode funcionar no laboratório como um local de transições de redes intensivas em conhecimento (ETZKOWITZ; LEYDESDORFF, 2000).

Matias-Pereira (2011) afirma que os relatórios e o discurso governamental sustentam que nos últimos anos a área de Ciência, Tecnologia e Inovação $(C, T \& I)$ passou a ser uma questão de Estado. $\mathrm{O}$ autor acrescenta que o foco nessa política foi orientado aos eixos estratégicos, definidos novos marcos regulatórios, criados novos instrumentos, formas de financiamento e aperfeiçoamento da gestão dos fundos setoriais, bem como um aumento dos recursos federais na área.

\subsection{O papel do Governo}

De acordo com Matias-Pereira (2011) a utilização intensiva da Ciência, Tecnologia e Inovação numa economia, permite a elevação da capacidade de competir, criando empreendimentos, empresas, empregos e marcas comerciais. Nesse contexto ressalta-se o papel do governo no sentido de fomentar investimentos possibilitando maior dinamismo e movimentação na economia regional ou nacional.

Dentre os diversos tipos de formas de investimento dos governos na área, é importante distinguir algumas denominações para compreender o direcionamento dos investimentos. $\mathrm{O}$ Ministério da Ciência, Tecnologia e Inovação trata das denominações: Ciência e Tecnologia (C\&T), Pesquisa e Desenvolvimento (P\&D) e Atividades científicas e Técnicas correlatas (ACTC). Os investimentos do governo em inovação são medidos através dos investimentos em C\&T. Esses investimentos são divididos entre P\&D e ACTC conforme demonstrado na Figura 2.

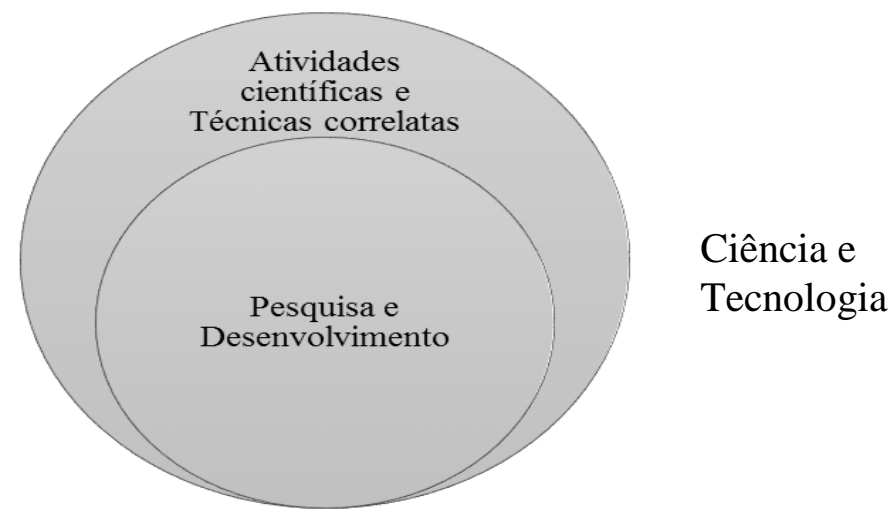

Figura 2 - Relação entre Ciência e Tecnologia, Pesquisa e Desenvolvimento e Atividades científicas e Técnicas correlatas.

Fonte: Adaptado de MCTI (2015). 
Com relação a definição e às atividades que compõem cada uma das áreas, as ACTC representam uma série de "ações que contribuem para a geração, difusão e aplicação do conhecimento científico, como serviços científicos e tecnológicos prestados por bibliotecas, museus de ciência, jardins botânicos, zoológicos, entre outros". Enquanto as atividades de P\&D tratam "as pesquisas básicas e aplicadas e o desenvolvimento experimental, ou seja, é o trabalho criativo voltado para aumentar o conhecimento da instituição ou empresa e criar novas aplicações" (MCTI, 2015).

\section{MATERIAIS E MÉTODOS}

A seguir são apresentados os procedimentos metodológicos realizados neste trabalho. Apresenta-se a classificação da pesquisa e o detalhamento de cada etapa realizada.

\subsection{Classificação da pesquisa}

Do ponto de vista de sua natureza, este trabalho é uma pesquisa aplicada uma vez que de acordo com Silva e Menezes (2005, p. 20) pesquisas deste tipo "objetivam gerar conhecimentos para aplicação prática e dirigidos à solução de problemas específicos”.

Quanto a forma de abordagem pode ser classificada como quantitativa, uma vez que foi realizada uma coleta de dados numéricos e utilizado o método de regressão da área de mineração de dados para análise e geração de resultados (GIL, 2008; SILVA; MENEZES, 2005).

Em relação aos objetivos, a pesquisa desenvolvida, classifica-se como descritiva. De acordo com Gil (2008), as pesquisas deste tipo visam descrever características de determinada população e estabelecer relações entre variáveis, inclusive por meio de técnicas padronizadas.

Do ponto de vista dos procedimentos técnicos realizados, é classificado como documental e experimental. Pelo fato de utilizar dados quantitativos disponibilizados pelo governo federal e de acordo com Gil (2008) documentos que já foram analisados, tais como relatórios de pesquisa e tabelas estatísticas podem ser caracterizados como documental. A caracterização como experimental deve-se ao fato de utilizar valores atuais como variáveis para a previsão de valores futuros (SILVA; MENEZES, 2005).

\subsection{Etapas da pesquisa}

O desenvolvimento da pesquisa pode ser dividido em quatro etapas. Após a obtenção dos dados a serem analisados, como uma primeira etapa foi realizada um pré-processamento dos dados para a eliminação de ruídos, ou seja, para retirar da análise dados que possam gerar resultados inconsistentes devido, principalmente, a existência de valores fora da curva (outliers).

Para isso, foi utilizada a técnica de agrupamento em casos onde verificou-se a necessidade para tal. $\mathrm{O}$ método $k$-means com o valor de $\mathrm{k}=3$ foi o algoritmo adotado no software Weka (THE UNIVERSITY OF WAIKATO, 2016) para o cumprimento desta etapa.

$\mathrm{Na}$ segunda etapa, é necessário escolher a função que será utilizada para o cálculo de regressão. Sendo assim, dentre as funções apresentadas pelo software CurveExpert (HYAMS, 2016), utilizado neste trabalho, foram consideradas as que apresentaram os cinco melhores coeficientes de determinação $\left(r^{2}\right)$. Entre essas cinco melhores funções, as polinomiais foram excluídas da análise. Por serem funções que apresentam um maior número de inflexões, essas funções apresentam um comportamento da curva mais fiel aos valores existentes, porém como o intuito é realizar uma previsão, é mais interessante que seja obtida uma curva com um comportamento mais genérico. 
Compondo a terceira etapa foram realizados os cálculos de regressão e previsão dos valores para os cinco anos seguintes (2014 a 2018). Por fim, na quarta e última etapa foi realizada a elaboração e análise dos gráficos gerados a partir dos cálculos realizados na etapa anterior.

\section{RESULTADOS E DISCUSSÃO}

Esta seção descreve a caracterização do objeto de estudo neste trabalho, além da apresentação dos resultados a partir da análise dos gráficos gerados.

\subsection{Caracterização do objeto de estudo}

Os valores analisados compreendem os investimentos em C\&T realizados pelo governo federal nas seguintes instituições: Ministério da Ciência, Tecnologia e Inovação (MCTI) Administração Central, Conselho Nacional de Desenvolvimento Científico e Tecnológico (CNPq), Comissão Nacional de Energia Nuclear (CNEN) e Agência Espacial Brasileira (AEB). O relatório utilizado compreende as instituições típicas de C\&T que são aquelas que realizam atividades de C\&T de modo permanente e organizado, tenham a ciência e/ou a tecnologia como atividade fim e objeto da aplicação da maior parte de seus recursos (MCTI, 2016).

Dentre as instituições vinculadas ao MCTI analisadas, além da administração geral do ministério, mais três instituições descritas a seguir:

- Agência Espacial Brasileira (AEB) - criada em 1994 é uma instituição responsável por formular, coordenar e executar a Política Espacial Brasileira que estabelece objetivos e diretrizes para os programas e projetos nacionais relativos à área espacial. Também é responsável pela coordenação geral do Sistema Nacional de Desenvolvimento das Atividades Espaciais, instituído para organizar a execução das atividades espaciais (AEB, 2017);

- Comissão Nacional de Energia Nuclear (CNEN) - foi criada em 1956 para desenvolver a política nacional de energia nuclear, sendo responsável por regular, licenciar e fiscalizar a produção e o uso de energia nuclear no país. Está focada em garantir os benefícios da energia nuclear a um número cada vez maior de brasileiros, buscando o uso cada vez mais amplo e seguro das técnicas do setor nuclear. Suas atividades estão distribuídas em duas grandes áreas: Pesquisa e Desenvolvimento e Radioproteção e Segurança. Possui unidades situadas em diferentes pontos do país com atividades voltadas para pesquisa, desenvolvimento e inovação entre outras (CNEN, 2017);

- Conselho Nacional de Desenvolvimento Científico e Tecnológico ( $\mathrm{CNPq})$ - Criado em 1951, tem como principais atribuições fomentar a pesquisa científica e tecnológica e incentivar a formação de pesquisadores. É um órgão de fomento à pesquisa, sendo responsável por participar da formulação, execução, acompanhamento e avaliação da difusão da Política Nacional de Ciência e Tecnologia (CNPQ, 2017);

- Ministério da Ciência, Tecnologia e Inovação (MCTI) - criado em 1985, concretizando um compromisso com a comunidade científica nacional. Estando sob sua competência assuntos como: política nacional de pesquisa científica, tecnológica e inovação, planejamento, coordenação, supervisão e controle das atividades da ciência e tecnologia e política de desenvolvimento de informática e automação (MCTI, 2017).

\subsection{Análise e apresentação dos resultados}

Os dados utilizados neste trabalho foram extraídos do Portal Brasileiro de Dados Abertos, que é uma ferramenta disponibilizada pelo governo federal para que os dados e informações públicas sejam acessíveis por toda a população. Os dados utilizados, especificamente, para esta 
pesquisa apresentam os dispêndios do governo federal em ciência e tecnologia (C\&T), aplicados pelo Ministério da Ciência, Tecnologia e Inovação (MCTI), por unidade orçamentária e atividade durante o período de 2000 a 2013. Tais valores referem-se ao investimento em ciência e tecnologia (C\&T) e estão divididos em pesquisa e desenvolvimento (P\&D) e atividades científicas e técnicas correlatas (ACTC).

Quadro 2 - Funções selecionadas para cada análise.

\begin{tabular}{|c|c|c|c|c|c|c|c|}
\hline & & $\begin{array}{l}\text { Logistic } \\
\text { Power }\end{array}$ & Rational Model & $\begin{array}{c}\text { Gaussian } \\
\text { Model }\end{array}$ & Sinusoidal & $\begin{array}{c}\text { Ratkowsky } \\
\text { Model }\end{array}$ & $\begin{array}{c}\text { Reciprocal } \\
\text { Quadratic }\end{array}$ \\
\hline & & $\begin{array}{c}t \\
+ \\
\underbrace{+}_{-1}\end{array}$ & 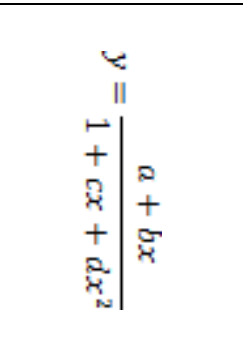 & 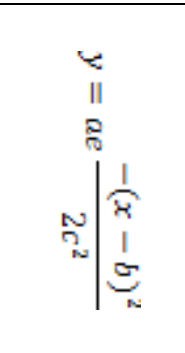 & 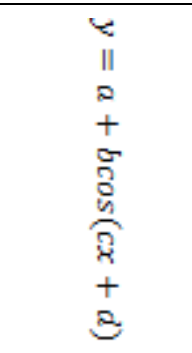 & 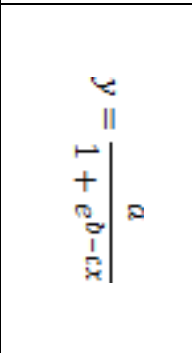 & 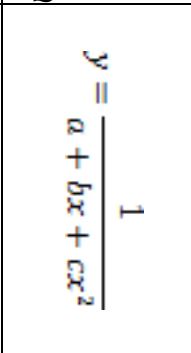 \\
\hline \multirow[t]{2}{*}{$\begin{array}{l}\text { MCTI - } \\
\text { Admin. } \\
\text { Central }\end{array}$} & P\&D & & & & $\begin{array}{l}\mathbf{a}=379,67 \\
\mathbf{b}=67,3 \\
\mathbf{c}=1,16 \\
\mathbf{d}=-388,78 \\
\mathbf{r}^{2}=0,28\end{array}$ & & \\
\hline & ACTC & $\begin{array}{l}\mathbf{a}=722,04 \\
\mathbf{b}=2005,76 \\
\mathbf{c}=-1140,08 \\
\mathbf{r}^{2}=0,65\end{array}$ & & & & & \\
\hline \multirow[t]{2}{*}{ CNPq } & P\&D & & & & & & $\begin{array}{l}\mathbf{a}=76,4 \\
\mathbf{b}=-0,08 \\
\mathbf{c}=0,00002 \\
\mathbf{r}^{2}=0,35\end{array}$ \\
\hline & ACTC & & & & & $\begin{array}{l}\mathbf{a}=321,65 \\
\mathbf{b}=338,36 \\
\mathbf{c}=0,17 \\
\mathbf{r}^{2}=0,99\end{array}$ & \\
\hline \multirow[t]{2}{*}{ CNEN } & P\&D & & & & $\begin{array}{l}\mathbf{a}=39,99 \\
\mathbf{b}=14,77 \\
\mathbf{c}=0,32 \\
\mathbf{d}=-395,25 \\
\mathbf{r}^{2}=0,94\end{array}$ & & \\
\hline & ACTC & & $\begin{array}{l}\mathbf{a}=2,45 \\
\mathbf{b}=-0,001 \\
\mathbf{c}=-0,00099 \\
\mathbf{d}=0,0000002 \\
\mathbf{r}^{2}=0,4\end{array}$ & & & & \\
\hline \multirow[t]{2}{*}{ AEB } & P\&D & $\begin{array}{l}\mathbf{a}=148,87 \\
\mathbf{b}=2004,06 \\
\mathbf{c}=-35458,78 \\
\mathbf{r}^{2}=0,73\end{array}$ & & & & & \\
\hline & ACTC & & & $\begin{array}{l}\mathbf{a}=105,68 \\
\mathbf{b}=2010,38 \\
\mathbf{c}=4,23 \\
\mathbf{r}^{2}=0,72\end{array}$ & & & \\
\hline
\end{tabular}

Fonte: Elaboração própria.

No Quadro 2 são apresentadas as funções selecionadas para o cálculo da previsão de investimentos para cada uma das instituições e do total investido pelo governo federal. 
Em três casos foi necessária a realização de pré-processamento dos dados. Para a análise do CNPq na área de P\&D foram excluídos os dados dos anos de 2012 e 2013, para a análise do CNPq na área de ACTC foi excluído o valor de 2011 e para a análise da AEB na área de ACTC foi excluído o valor do ano de 2011 por representarem, após a técnica de agrupamento, dados discrepantes em relação aos demais.

A Figura 3 apresenta os investimentos realizados pelo governo federal especificamente na área de Pesquisa e Desenvolvimento para as quatro instituições analisadas neste trabalho no período de 2000 a 2013 e o cálculo dos valores para os cinco anos seguintes.

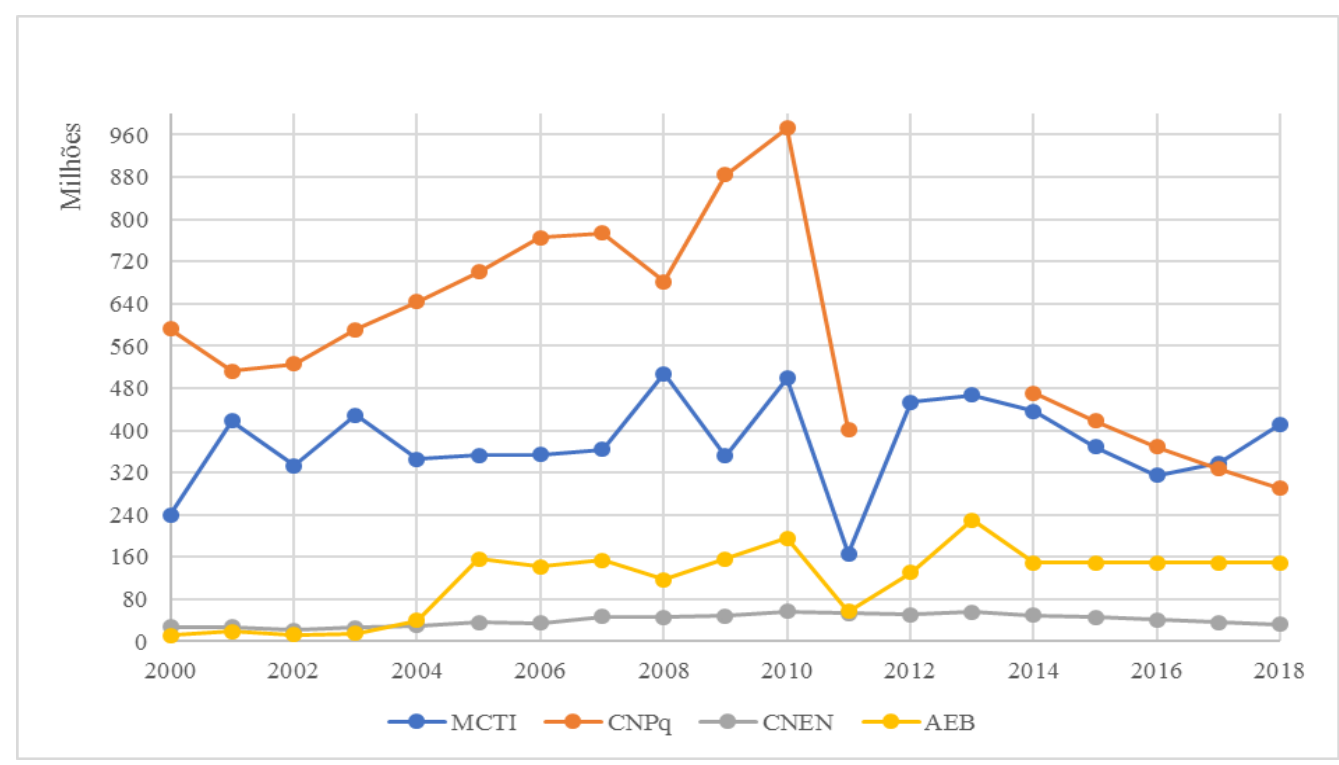

Figura 3 - Investimentos do governo federal em P\&D.

Fonte: Elaboração própria baseada em (MCTI, 2016).

A partir da Figura 3 é possível notar que no ano de 2010 todas as quatro instituições analisadas alcançaram um aumento considerável nos dispêndios do governo federal com P\&D. Outro dado identificado é uma grande queda em 2011, que afetou todas as instituições com exceção da CNEN que foi a instituição que manteve-se mais estável no período. Em relação a AEB, nota-se um aumento nos investimentos para esta instituição a partir de 2007, o que a levou a ultrapassar os investimentos repassados a CNEN.

Outro dado interessante é o fato do CNPq historicamente obter os maiores valores investidos dentre os órgãos analisados, apesar da retirada de dois valores referentes aos anos de 2012 e 2013 na etapa de pré-processamento. De acordo com os cálculos realizados pode chegar em 2018 ao valor mínimo de investimento já obtido, podendo receber um valor próximo a 290 milhões em investimentos. Ainda assim sendo um valor consideravelmente acima do investimento em AEB e, sobretudo, do CNEN que tende a receber um valor constante durante os anos, chegando em torno de 32 milhões em 2018.

Ainda em relação aos cálculos para o período entre os anos de 2014 e 2018, os investimentos não devem ultrapassar 415 milhões para as instituições. Para o CNEN e o CNPq é prevista uma queda no investimento, enquanto para a AEB os valores tendem a se manter estáveis no período previsto. Apenas para o MCTI há uma previsão de aumento nos investimentos até o ano de 2018.

A Figura 4 apresenta os investimentos realizados pelo governo federal especificamente na área de Atividades científicas e técnicas correlatas para as quatro instituições analisadas neste 
trabalho também no período de 2000 a 2013 e o cálculo dos valores repassados no período de 2014 a 2018.

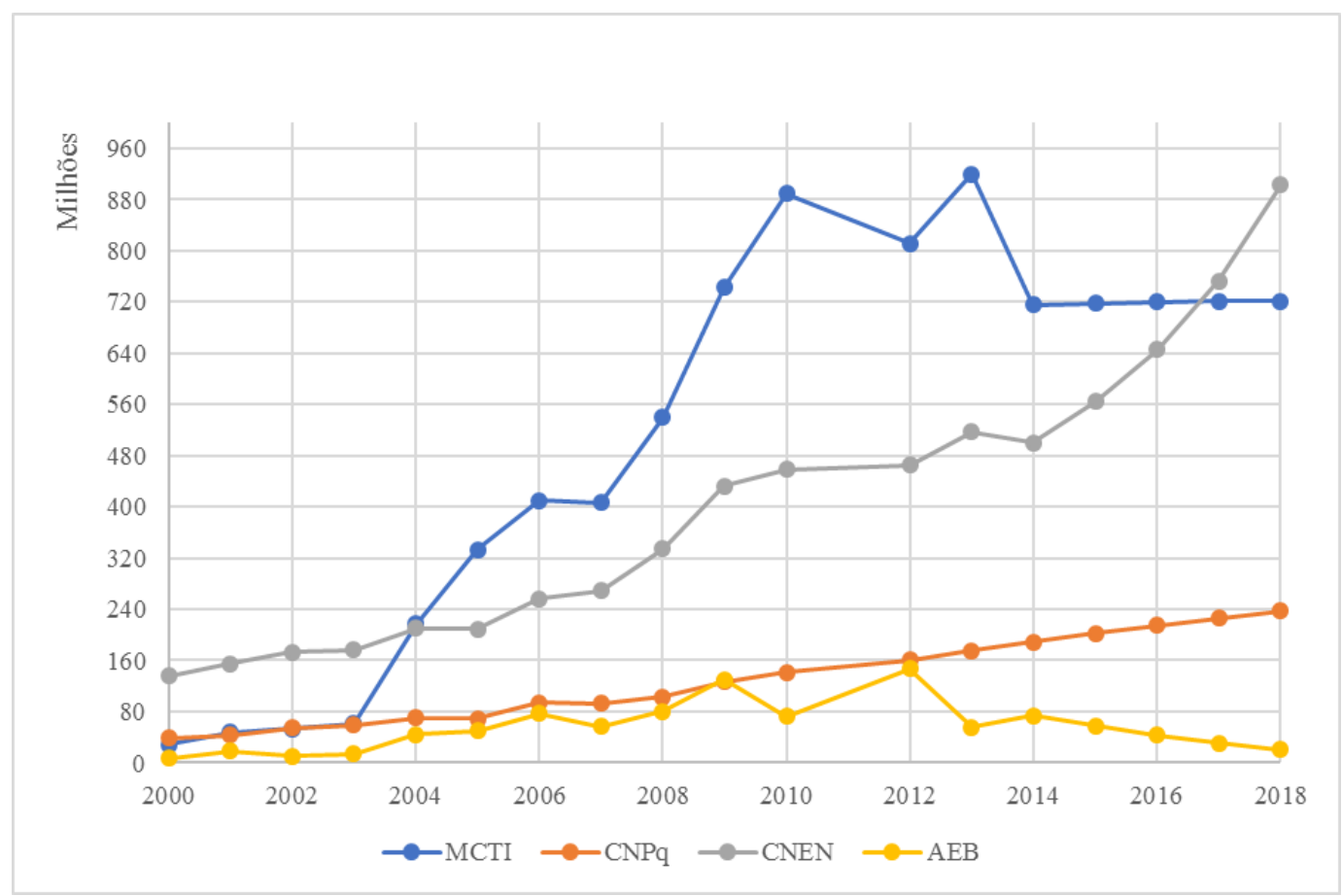

Figura 4 - Investimentos do governo federal em ACTC.

Fonte: Elaboração própria baseada em (MCTI, 2016).

O primeiro ponto a ser destacado quanto aos investimentos em ACTC apresentados na Figura 4 é que, em geral, sempre estiveram em crescimento no período compreendido entre os anos de 2000 a 2012, excetuando-se pequenas quedas pontuais. Importante frisar que os dados de 2011 foram retirados desta análise após a etapa de pré-processamento por representarem valores atípicos.

Com relação ao MCTI nota-se uma alta acentuada, sobretudo, entre os anos de 2003 e 2010 tornando-se a instituição a receber os maiores investimentos na área de ACTC. No entanto tal comportamento tende a ser modificado de acordo com os cálculos realizados para os anos de 2014 a 2018, quando os investimentos devem manter-se estáveis para esta instituição. A respeito da CNEN e do CNPq, a previsão é que os investimentos aumentem, mas para a CNEN a previsão apresenta resultados mais altos, podendo estes valores chegarem próximos a 900 milhões em 2018. Para o CNPq, apesar de aumentarem, estes valores não devem ultrapassar os 240 milhões até o ano de 2018.

O caso da AEB é o único dentre as instituições analisadas que deve ter os seus investimentos diminuídos durante o período previsto. A instituição deve receber em 2017, algo em torno de 310 milhões, e os valores devem cair, aproximadamente, 100 milhões em 2018.

A Figura 5 demonstra os valores repassados pelo governo federal às instituições analisadas no presente trabalho entre os anos de 2000 a 2013. Entre 2014 e 2018 são apresentadas as previsões dos investimentos na área de Ciência e tecnologia. 


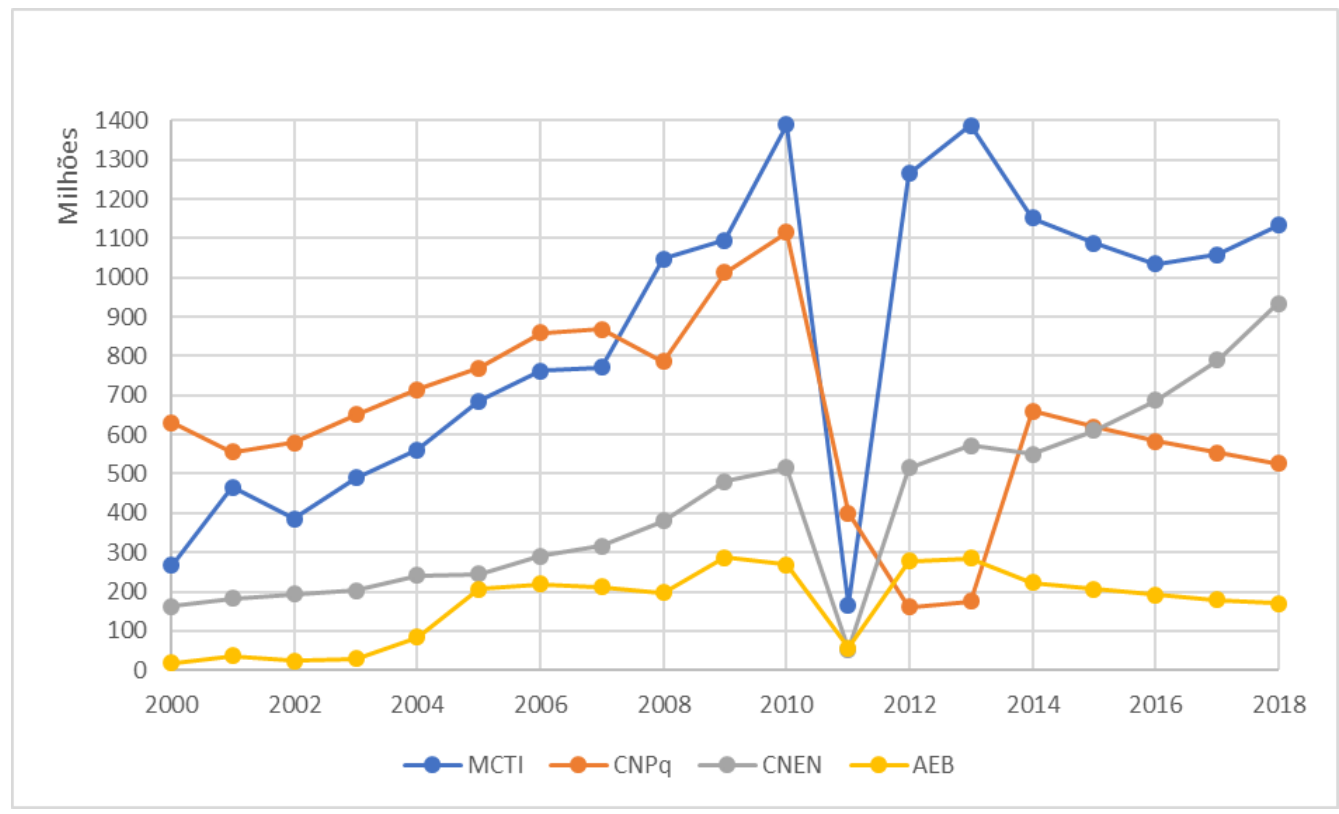

Figura 5 - Investimentos do governo federal em C\&T.

Fonte: Elaboração própria baseada em (MCTI, 2016).

A Figura 5 apresenta os valores investidos pelo governo federal em C\&T, ou seja, a soma dos investimentos em P\&D e em ACTC apresentados anteriormente. É importante destacar que a queda acentuada no ano de 2011 deve-se ao fato de que os valores correspondem apenas aos investimentos em $\mathrm{P} \& \mathrm{D}$, conforme explicado anteriormente. Outra observação, também já apresentada, refere-se aos valores do CNPq nos anos de 2012 e 2013.

A partir do gráfico apresentado na figura 5 pode-se observar que os investimentos para o CNPq e para o MCTI mostram-se mais próximos, entre os anos de 2001 a 2007, do que nas análises realizadas anteriormente. A partir da análise é possível identificar que os valores repassados a essas instituições separadamente, nivelaram-se quando apresentados em conjunto, representando os investimentos em C\&T.

Com relação a AEB, os investimentos mantiveram-se como os menores dentre as instituições analisadas, em função principalmente por receber os investimentos mais baixos em ACTC. Quanto ao CNEN, esse também encontra-se como um dos mais baixos em C\&T, apesar dos crescentes investimentos realizados em ACTC.

Sobre os investimentos realizados no período de 2014 a 2018, nota-se que o MCTI apresenta-se como a instituição com o maior repasse. O CNEN, de acordo com os cálculos, é a instituição que deve ter o maior aumento de investimento no período. Enquanto as outras duas instituições devem sofrer leve queda nos repasses do governo federal em C\&T.

Apesar do pré-processamento dos dados onde foram retirados ruídos (dados discrepantes), as quedas e elevações abruptas identificadas nos gráficos, sobretudo em 2011, contribuem para os valores previstos no período de 2014 a 2018.

\section{CONCLUSÃO}

Com abertura de mercado e aumento da competitividade a inovação tornou-se cada vez mais necessária e exigida. A partir da teoria da Tríplice hélice, que baseia-se na relação entre universidade, empresa e governo, torna-se claro o papel deste último componente: criar mecanismos 
para promover novas estruturas organizacionais, desenvolvimento econômico, áreas de atuação, bem como conduzir os processos de mudança.

O presente artigo analisou os investimentos em ciência e tecnologia $(\mathrm{C} \& \mathrm{~T})$ despendidos pelo governo federal entre os anos de 2000 a 2013, último ano em que o governo federal disponibilizou os dados, bem como fez uma previsão de investimentos para os cinco anos seguintes. Promovendo, assim, uma visão do comportamento do investimento do governo federal entre o período dos anos 2000 a 2018.

De acordo com Matias-Pereira (2011) a utilização intensiva da ciência e tecnologia e inovação numa economia, permite a elevação da capacidade de competir, criando empreendimentos, empresas, empregos e marcas comerciais. No estudo realizado por Luna et al. (2008) foi analisado que a trajetória dos investimentos públicos em C\&T foi marcada por forte instabilidade, principalmente na década de 90 e que somente a partir de 2000 os investimentos públicos passaram a crescer em regularidade.

Ao permitir maior conhecimento e acompanhamento da evolução de indicadores no tempo, como os valores investidos pelo governo federal em C\&T apresentados neste trabalho, seus resultados poderão ser utilizados de diversas formas como meio de agregar conhecimentos e explorar modos de financiamento previstos para os próximos cinco anos. Empresas, universidades e demais instituições podem estar mais atentas aos futuros investimentos gerados para as instituições analisadas.

De maneira geral, pode ser identificado que após períodos de crescimento e altas taxas de investimento e com base apenas em dados numéricos o que se prevê neste estudo é um período de aumento para o MCTI e a CNEN e leve queda para o CNPq e a AEB quanto aos investimentos do governo federal na área de C\&T.

\section{CONCLUSÂO}

A presente pesquisa apresenta algumas limitações. Primeiramente, é importante destacar que os dados utilizados, entre os anos de 2000 e 2013, compreendem os dados que foram disponibilizados pelo governo federal até o momento. Em razão disto, foram previstos os valores referentes aos anos 2014 a 2016, apesar de tais investimentos já terem sido realizados na prática para estes anos.

Outra limitação está relacionada a fatores externos que podem influenciar na previsão dos dispêndios do governo federal na área analisada. A forte crise política atual no país e a recessão econômica são fatores que podem influenciar consideravelmente nos valores apresentados após os cálculos de previsão realizados para os anos de 2014 a 2018. Tais fatores são extremamente variáveis e de difícil extração para inserção na previsão realizada. Em função disto, compreendem uma limitação da presente pesquisa.

Como propostas de trabalhos futuros pode-se obter os dados dos investimentos entre os anos de 2014 e 2016, assim que disponibilizados pelo governo federal, e confrontá-los com os valores apresentados no presente artigo. Além disso, uma outra abordagem pode utilizar de comparação dos investimentos brasileiros com outros países, como forma de verificar o nível de investimento brasileiro em C\&T em relação a países semelhantes. Por fim, existe a possibilidade de identificação e análise dos valores investidos em C\&T em relação ao PIB do país, como forma de identificar quanto é investido em pesquisa com relação a outras áreas. 


\section{REFERENCIAS}

AEB. Agência Espacial Brasileira. Institucional. Disponível em: <http://www.aeb.gov.br/institucional/sobre-a-aeb/> . Acesso em: 11 jan. 2017.

AMARAL, F. Aprenda Mineração de Dados: Teoria e prática. Rio de Janeiro: Alta Books Editora, 2016.

CALMANOVICI, C. E. A inovação, a competitividade e a projeção mundial das empresas brasileiras. Revista USP, v. 0, n. 89, p. 190-203, 1 maio 2011.

CARDOSO, O. N. P.; MACHADO, R. T. M. Gestão do conhecimento usando data mining estudo de caso na Universidade Federal de Lavras. 2008

CNEN. Comissão Nacional de Energia Nuclear. Institucional. Disponível em: <http://www.cnen.gov.br/>. Acesso em: 11 jan. 2017.

CNPQ. Conselho Nacional de Desenvolvimento Científico e Tecnológico. Institucional. Disponível em: <http://cnpq.br/apresentacao_institucional/>. Acesso em: 11 jan. 2017.

DAGNINO, R. A Relação Universidade-Empresa no Brasil e o “Argumento da Hélice Tripla”. Revista Brasileira de Inovação, v. 2, n. 2 jul/dez, p. 267-307, 17 ago. 2009.

ETZKOWITZ, H.; LEYDESDORFF, L. The dynamics of innovation: From National Systems and "mode 2" to a Triple Helix of university-industry-government relations. Research Policy, v. 29, n. 2, p. 109-123, 2000.

FAYYAD, U.; PIATETSKY-SHAPIRO, G.; SMYTH, P. From data mining to knowledge discovery in databases. AI magazine, v. 17, n. 3, p. 37, 1996.

GIL, A. C. Como elaborar projetos de pesquisa. São Paulo: Atlas, 2008.

GOLDSCHMIDT, R.; PASSOS, E. Data mining: um guia Prático. [s.l.] Gulf Professional Publishing, 2005.

HYAMS, D. G. CurveExpert Professional. Alabama, Estados Unidos.: [s.n.].

LUNA, F.; MOREIRA, S.; GONÇALVES, A. Financiamento à inovação. Políticas de incentivo à inovação tecnológica no Brasil. Brasília: Ipea, 2008.

MATIAS-PEREIRA, J. A gestão do sistema de proteção à propriedade intelectual no Brasil é consistente? Revista de Administração Pública, v. 45, n. 3, p. 567-590, jun. 2011.

MCTI. Aumenta o investimento em C\&T no Brasil. Disponível em: <http://www.mcti.gov.br/noticia//asset_publisher/epbV0pr6eIS0/content/aumenta-o-investimento-em-c-t-no-

brasil;jsessionid=BF0B6A9337FF0D6064C697804477A8F4>. Acesso em: 23 dez. 2016.

MCTI. Brasil: Dispêndios do governo federal em ciência e tecnologia (C\&T), aplicados pelo MCTI, por unidade orçamentária e atividade, 2000-2014. <http://www.mct.gov.br/index.php/content/view/5410/Brasil_Dispendios_do_governo_federal_em_ciencia_e_tecnologi a_C_T_sup_1_2_sup_aplicados_pelo_Ministerio_da_Ciencia_Tecnologia_e_Inovacao_MCTI_por_unidade_orcament aria_e_atividade.html>. Acesso em: 3 jan. 2017.

MCTI. Ministério da Ciência, Tecnologia, Inovações e Comunicações. Institucional. Disponível em: <http://www.mcti.gov.br/>. Acesso em: 11 jan. 2017.

PEREIRA NETO, A.; GALlindO, F.; CRUZ, S. R. DA. O Programa de Apoio à Pesquisa em Empresas e o Rio Inovação: uma avaliação preliminar. Inteligência empresarial, v. 1, n. 21, p. 4-12, 2004

SANTOS, A. C. DE S. G. DOS; MENEZES, T. DE P.; HORA, H. R. M. DA. Análise do perfil de aluno e egresso de cursos técnicos por meio de data mining: estudo de caso no Instituto Federal Fluminense. \#Tear: Revista de Educação, Ciência e Tecnologia, v. 3, n. 1, 19 jun. 2014.

SILVA, E. L. DA; MENEZES, E. M. Metodologia da pesquisa e elaboração de dissertação. UFSC, Florianópolis, 4a. edição, 2005.

SILVA, R. M. DA et al. Percepção dos discentes em relação aos docentes através da aplicação de técnicas e métodos de mineração de dados. . In: XXXVIIII CONGRESSO BRASILEIRO DE EDUCAÇÃO EM ENGENHARIA. Fortaleza, CE, Brasil: 2010Disponível <http://www.abenge.org.br/CobengeAnteriores/2010/artigos/549.doc> 
THE UNIVERSITY OF WAIKATO. Weka. Hamilton, Nova Zelândia: [s.n.].

THERG-BRAZIL. Triple Helix Research Group - THERG-Brazil, 2013. Disponível em: <www.triplehelix.uff.br/>. Acesso em: 16 dez. 2016

TIDD, J.; BESSANT, J. R. Managing innovation: integrating technological, market and organizational change. Fifth edition ed. Chichester, West Sussex, United Kingdom: John Wiley \& Sons, 2013.

WITTEN, I. H.; FRANK, E. Data mining: practical machine learning tools and techniques. 2. ed ed. San Francisco, Calif.: Kaufmann [u.a.], 2005. 


\section{ANEXO}

Dispêndios do governo federal em C\&T, aplicados pelo Ministério da Ciência, Tecnologia e Inovação (MCTI), por unidade orçamentária e atividade, 2000-2013.

(em milhões de R $\$$ correntes)

\begin{tabular}{|c|c|c|c|c|c|}
\hline \multicolumn{2}{|c|}{ Ano/Atividade } & \multirow{2}{*}{$\begin{array}{l}\text { Ministério da Ciência, } \\
\text { Tecnologia e Inovação - } \\
\text { Administração Central } \\
267.8\end{array}$} & \multirow{2}{*}{$\begin{array}{c}\begin{array}{c}\text { Conselho Nacional de } \\
\text { Desenvolvimento Científico } \\
\text { e Tecnológico - CNPq }\end{array} \\
\mathbf{6 3 0 . 7 6}\end{array}$} & \multirow{2}{*}{$\begin{array}{r}\begin{array}{c}\text { Comissão Nacional } \\
\text { de Energia Nuclear } \\
\text { - CNEN }\end{array} \\
162.49\end{array}$} & \multirow{2}{*}{$\begin{array}{r}\text { Agência Espacial } \\
\text { Brasileira - AEB } \\
18.27\end{array}$} \\
\hline \multirow{3}{*}{2000} & C\&T & & & & \\
\hline & $\mathrm{P} \& \mathrm{D}$ & 239.45 & 592.22 & 27.14 & 11.66 \\
\hline & ACTC & 28.34 & 38.53 & 135.34 & 6.61 \\
\hline \multirow{3}{*}{2001} & C\&T & 465.55 & 555.95 & 182.45 & 37.36 \\
\hline & $\mathrm{P} \& \mathrm{D}$ & 418.41 & 512.28 & 27.75 & 19.02 \\
\hline & ACTC & 47.14 & 43.67 & 154.69 & 18.33 \\
\hline \multirow{3}{*}{2002} & C\&T & 386.12 & 579.64 & 194.28 & 23.05 \\
\hline & P\&D & 332.99 & 525.48 & 21.9 & 12.33 \\
\hline & ACTC & 53.12 & 54.15 & 172.38 & 10.72 \\
\hline \multirow{3}{*}{2003} & C\&T & 489.93 & 650.83 & 202.58 & 29.1 \\
\hline & $P \& D$ & 429,0 & 591.43 & 25.87 & 14.73 \\
\hline & ACTC & 60.92 & 59.39 & 176.7 & 14.36 \\
\hline \multirow{3}{*}{2004} & C\&T & 561.81 & 714.17 & 241,0 & 83.94 \\
\hline & $\mathrm{P} \& \mathrm{D}$ & 345.07 & 643.97 & 30.54 & 39.36 \\
\hline & ACTC & 216.73 & 70.2 & 210.45 & 44.58 \\
\hline \multirow{3}{*}{2005} & C\&T & 686.09 & 768.93 & 244.82 & 205.79 \\
\hline & $\mathrm{P} \& \mathrm{D}$ & 352.45 & 700.29 & 35.85 & 156.34 \\
\hline & ACTC & 333.64 & 68.63 & 208.96 & 49.44 \\
\hline \multirow{3}{*}{2006} & C\&T & 763.11 & 859.82 & 290.74 & 218.83 \\
\hline & $\mathrm{P} \& \mathrm{D}$ & 353.81 & 765.79 & 34.39 & 141.51 \\
\hline & ACTC & 409.3 & 94.02 & 256.34 & 77.32 \\
\hline \multirow{3}{*}{2007} & C\&T & 770.67 & 867.8 & 315.8 & 211.19 \\
\hline & P\&D & 364.31 & 774.45 & 47.4 & 154.32 \\
\hline & ACTC & 406.36 & 93.35 & 268.4 & 56.86 \\
\hline \multirow{3}{*}{2008} & C\&T & 1047.04 & 785.1 & 380.5 & 197.99 \\
\hline & P\&D & 507.94 & 681.64 & 46.09 & 117.17 \\
\hline & ACTC & 539.09 & 103.45 & 334.4 & 80.81 \\
\hline \multirow{3}{*}{2009} & C\&T & 1095.31 & 1011.97 & 480.79 & 286.95 \\
\hline & P\&D & 352.23 & 884.84 & 48.43 & 156.95 \\
\hline & ACTC & 743.07 & 127.13 & 432.35 & 129.99 \\
\hline \multirow{3}{*}{2010} & C\&T & 1388.77 & 1114.29 & 515.14 & 268.25 \\
\hline & P\&D & 499.55 & 973.11 & 57.21 & 195.27 \\
\hline & ACTC & 889.21 & 141.17 & 457.92 & 72.98 \\
\hline \multirow{3}{*}{2011} & C\&T & 243.05 & 1213.4 & 52.76 & 522.51 \\
\hline & P\&D & 166.12 & 400.44 & 52.76 & 57.22 \\
\hline & ACTC & 76.93 & 812.96 & - & 465.28 \\
\hline \multirow{3}{*}{2012} & C\&T & 1265.5 & 1515.85 & 515.46 & 278.05 \\
\hline & P\&D & 453.83 & 1355.48 & 50.83 & 130.82 \\
\hline & ACTC & 811.66 & 160.37 & 464.62 & 147.23 \\
\hline \multirow{3}{*}{2013} & C\&T & 1387.29 & 2034.55 & 572.57 & 285.85 \\
\hline & $P \& D$ & 467.62 & 1859.27 & 55.83 & 230.28 \\
\hline & ACTC & 919.67 & 175.28 & 516.73 & 55.57 \\
\hline
\end{tabular}

Fonte: (MCTI, 2016). 